EGU2020-3472

https://doi.org/10.5194/egusphere-egu2020-3472

EGU General Assembly 2020

(c) Author(s) 2020. This work is distributed under

the Creative Commons Attribution 4.0 License.

\title{
Assessment of Air Pollution in the Middle East Using Reanalyses Products and High-resolution WRF-Chem Simulations
}

\author{
Alexander Ukhov ${ }^{1}$, Suleiman Mostamandi ${ }^{1}$, Johannes Flemming ${ }^{2}$, Arlindo DaSilva ${ }^{3}$, Nick Krotkov ${ }^{3}$, \\ Can Li ${ }^{3}$, Yasser Alshehri ${ }^{1}$, Anatolii Anisimov ${ }^{1,4}$, Vitali Fioletov ${ }^{5}$, Chris McLinden ${ }^{5}$, Illia Shevchenko ${ }^{1}$, \\ and Georgiy Stenchikov ${ }^{1}$ \\ ${ }^{1}$ King Abdullah University of Science and Technology, Division of Physical Sciences and Engineering, Thuwal, Saudi Arabia \\ (alexander.ukhov@kaust.edu.sa) \\ ${ }^{2}$ European Centre for Medium-Range Weather Forecasts, Reading, UK \\ ${ }^{3}$ NASA Goddard Space Flight Center, Greenbelt, MD, USA \\ ${ }^{4}$ Marine Hydrophysical Institute, Russian Academy of Sciences, Sevastopol, Russia \\ ${ }^{5}$ Air Quality Research Division, Environment Canada, Toronto, ON, Canada
}

The Middle East is notorious for high air pollution that affects both air-quality and regional climate. The Middle East generates about 30\% of world dust annually and emits about $10 \%$ of anthropogenic $\mathrm{SO}_{2}$. In this study we use Modern-Era Retrospective analysis for Research and Applications v.2 (MERRA-2), Copernicus Atmosphere Monitoring Service Operational Analysis (CAMS-OA) data assimilation products, and a regional Weather Research and Forecasting model (10 km resolution) coupled with Chemistry (WRF-Chem) to evaluate natural and anthropogenic air pollution in the ME. The $\mathrm{SO}_{2}$ anthropogenic emissions used in WRF-Chem are updated using the independent satellite $\mathrm{SO}_{2}$ emission dataset obtained from the Ozone Monitoring Instrument (OMI) observations onboard NASA EOS Aura satellite. Satellite and ground-based aerosol optical depth (AOD) observations, as well as Particulate Matter (PM) and $\mathrm{SO}_{2}$ in situ measurements for 2015-2016, were used for validation and model evaluation.

Although aerosol fields in regional WRF-Chem and global assimilation products are quite consistent, WRF-Chem, due to its higher spatial resolution and novel $\mathrm{OMI} \mathrm{SO}_{2}$ emissions, is preferable for analysis of regional air-quality over the ME. We found that conventional emission inventories (EDGAR-4.2, MACCity, and HTAP-2.2) have uncertainties in the location and magnitude of $\mathrm{SO}_{2}$ sources in the ME and significantly underestimate $\mathrm{SO}_{2}$ emissions in the Arabian Gulf. CAMS reanalysis tends to overestimate $\mathrm{PM}_{2.5}$ and underestimate $\mathrm{PM}_{10}$ concentrations. In the coastal areas, MERRA2 underestimates sulfate and tends to overestimate sea salt concentrations. The WRF-Chem's PM background concentrations exceed the World Health Organization (WHO) guidelines over the entire ME. The major contributor to PM ( 75-95\%) is mineral dust. In the ME urban centers and near oil recovery fields, non-dust aerosols (primarily sulfate) contribute up to $26 \%$ into $\mathrm{PM}_{2.5}$. The contribution of sea salt into $\mathrm{PM}$ can rich up to $5 \%$. The contribution of organic matter into PM prevails over black carbon. $\mathrm{SO}_{2}$ surface concentrations in major ME cities frequently exceed European air-quality limits. 
